EPJ Web of Conferences 73, 03008 (2014)

DOI: $10.1051 /$ epjconf/20147303008

(C) Owned by the authors, published by EDP Sciences, 2014

\title{
Onia and onia-like states at LHCb
}

Giovanni Passaleva ${ }^{a}$ On behalf of the LHCb collaboration

Istituto Nazionale di Fisica Nucleare, Sezione di Firenze, Florence, Italy

\begin{abstract}
We present recent LHCb results of studies on the production of $\mathrm{J} / \psi$ and $\chi_{\mathrm{c}}$ charmonium states, of $\Upsilon$ bottomonia and on $\mathrm{J} / \psi$ polarization in proton-proton collisions at $\sqrt{s}=8 \mathrm{TeV}$. Results on $\mathrm{J} / \psi$ production in proton-lead collisions are also presented.
\end{abstract}

\section{Introduction}

The mechanism of heavy quarkonium production is still not completely understood theoretically. The theoretical framework to approach this subject is represented by the non-relativistic QCD (NRQCD) models $[1,2]$ that include colour-singlet (CS) and colour-octet (CO) amplitude calculations. The CS model (CSM) [3, 4] underestimates the observed cross-section for single $\mathrm{J} / \psi$ production at high transverse momentum. To resolve this discrepancy, the CO mechanism [5] was introduced. More recent higher-order calculations [6-8], reduce the discrepancy between CSM and experimental data [9]. However, none of these approaches can reproduce in a consistent way the available experimental results on both cross-section and polarisation (see [9] and references therein). Charmonium is also produced from b-hadron decays. In this case QCD predictions are based on the Fixed-Order-Next-to-Leading-Log (FONLL) formalism $[10,11]$. In the following, results from heavy quarkonium production at $\mathrm{LHCb}$ will be compared with various theoretical models to provide direct tests of the underlying production mechanism.

\section{Results on production of heavy quarkonia}

\subsection{Production of $\mathrm{J} / \psi$ and $\Upsilon(\mathrm{nS})$ mesons}

The double differential production cross-sections as function of the meson rapidity $y$ and transverse momentum $\mathrm{p}_{\mathrm{T}}$ of prompt $\mathrm{J} / \psi$ and $\Upsilon(\mathrm{nS})$ mesons produced at the pp interaction point, either directly or via feed-down from higher mass quarkonium states, are measured at $\sqrt{s}=8 \mathrm{TeV}$ [12] with the $\mathrm{LHCb}$ detector [13]. The measurements are performed in the range $2.0<y<4.5$ and $\mathrm{p}_{\mathrm{T}}<14 \mathrm{GeV} / \mathrm{c}$ $\left(\mathrm{p}_{\mathrm{T}}<15 \mathrm{GeV} / \mathrm{c}\right)$ for the $\mathrm{J} / \psi(\Upsilon(\mathrm{nS}))$. The fraction of $\mathrm{J} / \psi$ coming from b-hadron decays (abbreviated as "from b" in the following) is also measured in the same fiducial region. The data samples correspond to an integrated luminosity of about $51 \mathrm{pb}^{-1}$ for the $\Upsilon(\mathrm{nS})$ and to about $18 \mathrm{pb}^{-1}$ for the $\mathrm{J} / \psi$. The $\mathrm{J} / \psi$ and $\Upsilon(\mathrm{nS})$ are reconstructed through their decay into a pair of muons $[14,15]$. The invariant

\footnotetext{
ae-mail: giovanni.passaleva@fi.infn.it
}

This is an Open Access article distributed under the terms of the Creative Commons Attribution License 4.0, which permits unrestricted use, distribution, and reproduction in any medium, provided the original work is properly cited. 

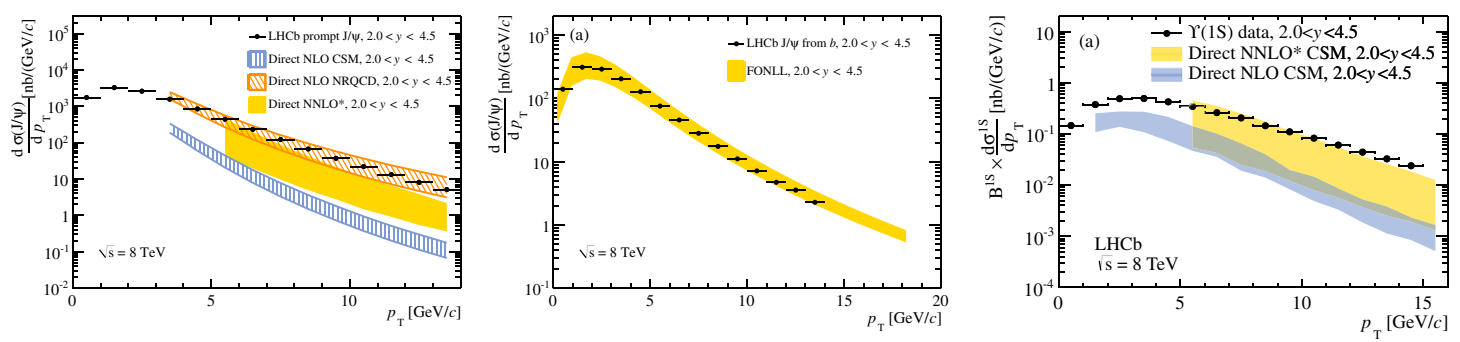

Figure 1. Left: comparison of the differential cross-section for prompt unpolarized $\mathrm{J} / \psi$ as a function of $\mathrm{p}_{\mathrm{T}}$ with three theoretical models: NLO NRQCD [19, 20] (orange diagonal shading), NNLO* CSM [7, 8] (solid yellow) and NLO CSM [21] (blue vertical shading). Middle: differential cross-section for $J / \psi$ from $b$ as a function of $\mathrm{p}_{\mathrm{T}}$ for $2.0<y<4.5$ compared with the FONLL prediction [10,11]. Right: comparison of the differential production cross-sections times dimuon branching fractions for $\Upsilon(1 \mathrm{~S})$, mesons as a function of $\mathrm{p}_{\mathrm{T}}$ with direct production in an NNLO* CSM [7] (solid yellow) and an NLO CSM [21] (blue vertical shading) model.

mass resolution is on average $13.5 \mathrm{MeV} / \mathrm{c}^{2}$ for the $\mathrm{J} / \psi$ and $43 \mathrm{MeV} / \mathrm{c}^{2}$ for the $\Upsilon(1 \mathrm{~S})$ meson. The mesons are assumed unpolarized, given the small polarisation measured for the $\mathrm{J} / \psi$ by LHCb [16] (discussed in Sect. 3) and ALICE [17], and for the $\Upsilon(\mathrm{nS})$ by CMS [18]. The measured differential cross-sections for prompt $\mathrm{J} / \psi$ mesons as a function of $\mathrm{p}_{\mathrm{T}}$ are compared in Fig. 1 (left) to three theoretical models: a NLO NRQCD calculation [19, 20]; NNLO* CSM [7, 8] (NNLO* indicates that the calculation at NNLO neglects part of the logarithmic terms) and an NLO CSM [21]. The theoretical models do not take into account the feed-down contribution which can be as large as $20 \%$ as in the case of $\chi_{\mathrm{c}} \rightarrow \mathrm{J} / \psi \gamma$ radiative decays [22]. Allowing for this contribution, NNLO* CSM and the NLO NRQCD models are in reasonable agreement with data while the CSM at NLO underestimates the cross-section by an order of magnitude. In Fig. 1 (middle) the differential cross-section for J/ $\psi$ from $\mathrm{b}$ as a function of $\mathrm{p}_{\mathrm{T}}$ at $\sqrt{s}=8 \mathrm{TeV}$ is compared to the FONLL predictions. Very good agreement is observed. The integrated cross-section for $\mathrm{J} / \psi$ from $\mathrm{b}$ production in the defined fiducial region is determined to be $\sigma(\mathrm{J} / \psi$ from $\mathrm{b} ; \mathrm{pT}<14 \mathrm{GeV} / \mathrm{c} ; 2.0<y<4.5)=1.28 \pm 0.01$ (stat.) \pm 0.11 (sys.) $\mu \mathrm{b}$ [12]. Extrapolating the previous result to the full kinematic region using Pythia [23], and using the inclusive $\mathrm{b} \rightarrow \mathrm{J} / \psi \mathrm{X}$ branching fraction, we derive the total $\mathrm{b} \overline{\mathrm{b}}$ production cross-section at $\sqrt{\mathrm{s}}=$ $8 \mathrm{TeV}, \sigma(\mathrm{pp} \rightarrow \mathrm{b} \overline{\mathrm{bX}})=298 \pm 2$ (stat.) \pm 36 (sys.) $\mu \mathrm{b}$ [12]. The measured cross-sections times dimuon branching fractions for the three $\Upsilon$ states are found to be in reasonable agreement with the NNLO* CSM [7] while the CSM at NLO [21] underestimates the cross-sections by an order of magnitude, as shown for the $\Upsilon(1 \mathrm{~S})$ meson in Fig. 1 (right).

\subsection{Production of $\mathrm{J} / \psi$ mesons in proton-lead collisions}

$\mathrm{LHCb}$ has recently published results on production of $\mathrm{J} / \psi$ mesons in proton-lead collisions at $\sqrt{s_{N N}}=$ $5 \mathrm{TeV}$ [24]. Data were collected with a proton beam energy of $4 \mathrm{TeV}$ and a lead beam energy of $1.58 \mathrm{TeV}$ per nucleon. The directions of the proton and lead beams were swapped to produce both $\mathrm{pPb}$ and $\mathrm{Pbp}$ collisions. The data samples correspond to integrated luminosities of $0.75 \mathrm{nb}^{-1} \mathrm{of} \mathrm{pPb}$ and $0.30 \mathrm{nb}^{-1}$ of Pbp collisions. The double-differential cross-section is measured for prompt $\mathrm{J} / \psi$ and $\mathrm{J} / \psi$ from $\mathrm{b}$ in bins of $y$ and $\mathrm{p}_{\mathrm{T}}$. From these measurements we derive the nuclear modification factor $R_{p P b}\left(y ; \sqrt{s_{N N}}\right)=\frac{1}{A} \frac{d \sigma_{p A}}{d y}\left(y ; \sqrt{s_{N N}}\right) / \frac{d \sigma_{p p}}{d y}\left(y ; \sqrt{s_{N N}}\right)$ and the forward-backward production ratio $R_{F B}\left(y ; \sqrt{s_{N N}}\right)=R_{p P b}\left(+|y| ; \sqrt{s_{N N}}\right) / R_{p P b}\left(-|y| ; \sqrt{s_{N N}}\right)$ whose distributions are shown in Fig. 2 and compared to theoretical predictions [25-27]. Within the available statistics good agreement is observed. 

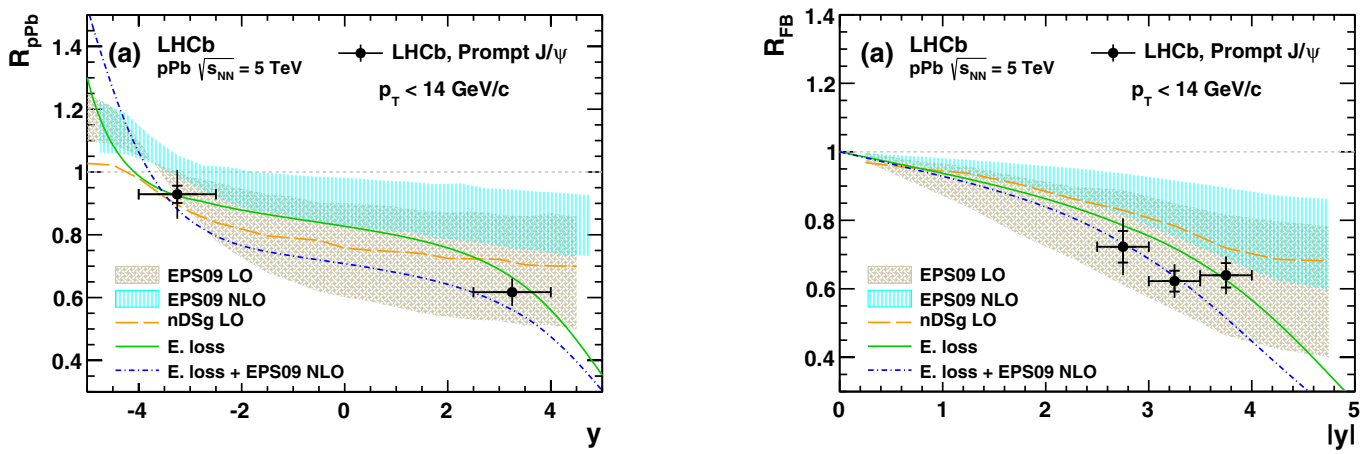

Figure 2. Distributions of $R_{p P b}$ (left) and $R_{F B}$ (right) as a function of $y$ for prompt $\mathrm{J} / \psi$ mesons produced in protonlead collisions, together with the theoretical predictions from (yellow dashed line and brown band) [25], (blue band) [26], and (green solid and blue dash-dotted lines) [27].
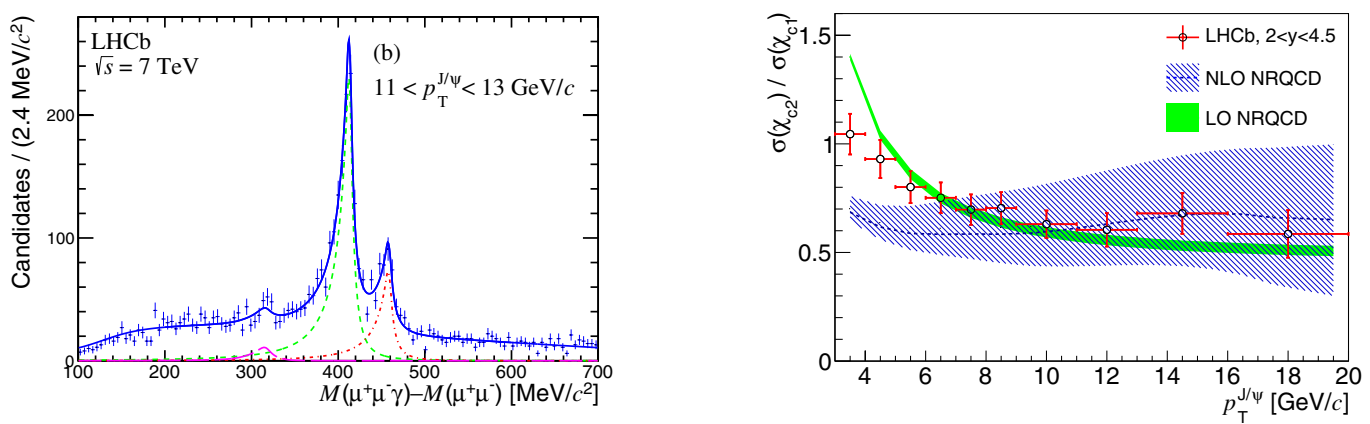

Figure 3. Left: $\chi_{\mathrm{c}}-\mathrm{J} / \psi$ mass difference spectrum in the range $11-13 \mathrm{GeV} / \mathrm{c}$. The results of the fit are also shown, with the total fitted function (blue solid curve), the $\chi_{\mathrm{c} 1}$ signal (green dashed curve), the $\chi_{\mathrm{c} 2}$ signal (red dot-dashed curve) and the $\chi_{\mathrm{co}}$ signal (purple long-dashed curve). Right: comparison of the LHCb results (with total uncertainty) with the NLO NRQCD calculation from [28] (blue shading) and the LO NRQCD calculation of [29] (solid green). The $\mathrm{LHCb}$ results are obtained assuming the $\chi_{\mathrm{c} 1}$ mesons are produced unpolarized.

\subsection{Production of $\chi_{c}$ mesons}

The ratio of $\chi_{\mathrm{c} 2}$ to $\chi_{\mathrm{c} 1}$ production cross-sections is sensitive to charmonium production models. $\mathrm{LHCb}$ has recently published a measurement of the ratio of prompt unpolarized $\chi_{\mathrm{c} 2}$ to $\chi_{\mathrm{c} 1}$ production crosssections at $\sqrt{s}=7 \mathrm{TeV}$ in the rapidity range $2.0<y<4.5$ as a function of the $\mathrm{J} / \psi \mathrm{p}_{\mathrm{T}}$ from 3 to $20 \mathrm{GeV} / \mathrm{c}$ [30]. The data sample corresponds to an integrated luminosity of $1.0 \mathrm{fb}^{-1}$. We use the radiative decay $\chi_{\mathrm{c}} \rightarrow \mathrm{J} / \psi\left(\rightarrow \mu^{+} \mu^{-}\right) \gamma$, and only photons converted in $\mathrm{e}^{+} \mathrm{e}^{-}$pairs in the detector material are used. The $\chi_{\mathrm{c}}-\mathrm{J} / \psi$ mass difference spectrum is shown in Fig. 3. A peak in correspondence to the $\chi_{\mathrm{c} 0}$ state is visible, which represents the first evidence of this state at a high-energy hadron collider, with a statistical significance of $4.3 \sigma$. In Fig. 3 the measured ratio $\sigma\left(\chi_{\mathrm{c} 2}\right) / \sigma\left(\chi_{c 1}\right)$ as a function of the $\mathrm{J} / \psi \mathrm{p}_{\mathrm{T}}$ is compared with the NLO NRQCD calculation of [28] and with the LO NRQCD calculation of [29]. Reasonable agreement with the theoretical predictions is observed for $\mathrm{p}_{\mathrm{T}}>4 \mathrm{GeV} / \mathrm{c}$.

\section{Measurement of $\mathrm{J} / \psi$ polarization}

$\mathrm{LHCb}$ has recently published a full angular analysis of the prompt $\mathrm{J} / \psi$ decays into muon pairs measuring the three polarization parameters $\lambda_{\theta}, \lambda_{\phi}$ and $\lambda_{\theta \phi}$ [31]. The data analysed correspond to $0.37 \mathrm{fb}^{-1}$ of $\mathrm{pp}$ 


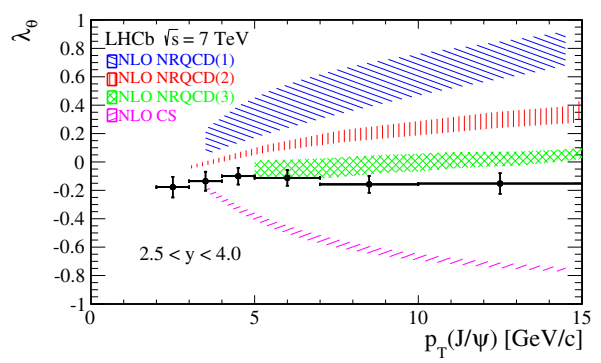

Figure 4. Comparison of LHCb prompt $\mathrm{J} / \psi$ polarization measurements of $\lambda_{\theta}$ with direct NLO CS (magenta diagonal lines) [32] and three different NLO NRQCD (blue diagonal lines (1) [32], red vertical lines (2) [33] and green hatched (3) [34]) predictions as a function of the $\mathrm{p}_{\mathrm{T}}$ of the $\mathrm{J} / \psi$ meson in the range $2.5<y<4.0$ in the helicity frame.

collisions at $\sqrt{s}=7 \mathrm{TeV}$. The polarization parameters are measured in the helicity frame [12] in bins of $y$ and $\mathrm{p}_{\mathrm{T}}$ in the range $2.0<y<4.5$ and $2<\mathrm{p}_{\mathrm{T}}<15 \mathrm{GeV} / \mathrm{c}$. A small longitudinal polarization $\lambda_{\theta}$ is observed while parameters $\lambda_{\phi}$ and $\lambda_{\theta \phi}$ are found to be compatible with zero. The measured $\lambda_{\theta}$ as a function of $\mathrm{p}_{\mathrm{T}}$ and integrated over $y$ is compared with three different NLO NRQCD calculations [3234] and with the NLO CSM prediction [32] in Fig. 4. The CSM calculation does not reproduce the experimental values. Among the NLO NRQCD, those that include the contributions from feed-down $([33,34])$ are in better agreement with data with a preference for the calculation in [34].

\section{Conclusions}

$\mathrm{LHCb}$ has performed a wealth of measurements on quarkonium production at different center-of-mass energies, and many more are underway. They allow an in depth comparison with theoretical models. A simple CSM is disfavoured by the data, while a combination of CS and CO, as implemented in the NRQCD formalism, or CS improved by QCD corrections, provide a good description of prompt quarkonium production. Charmonium production from b-hadron decays is very well reproduced by FONLL calculations. The predictions of NLO NRQCD calculations are also in reasonable agreement with $\mathrm{J} / \psi$ polarization measurements. Other results discussed in these proceedings, such as those on $\chi_{\mathrm{c}}$ production and on $\mathrm{J} / \psi$ production in proton-lead collisions at $\sqrt{s_{N N}}=5 \mathrm{TeV}$ also show reasonable agreement with the theoretical predictions, though with reduced statistical precision. The analysis of the full $\mathrm{LHCb}$ dataset is in progress and will reduce the statistical uncertainty giving access to more precise comparisons with the theoretical models.

\section{References}

[1] W. Caswell, G. Lepage, Phys. Lett. B 167, 437 (1986)

[2] G.T. Bodwin, E. Braaten, G.P. Lepage, Phys. Rev. D 51, 1125 (1995), hep-ph/9407339

[3] V. Kartvelishvili, A. Likhoded, S. Slabospitsky, Sov. J. Nucl. Phys. 28, 678 (1978)

[4] R. Baier, R. Rückl, Physics Letters B 102, 364 (1981)

[5] E. Braaten, S. Fleming, Phys. Rev. Lett. 74, 3327 (1995), hep-ph/9411365

[6] B. Gong, J.X. Wang, Phys. Rev. Lett. 100, 232001 (2008), 0802.3727

[7] P. Artoisenet, J.M. Campbell, J. Lansberg, F. Maltoni, F. Tramontano, Phys. Rev. Lett. 101, 152001 (2008), 0806.3282

[8] J. Lansberg, Eur. Phys. J. C 61, 693 (2009), 0811.4005 
[9] N. Brambilla, S. Eidelman, B. Heltsley, R. Vogt, G. Bodwin et al., Eur. Phys. J. C 71, 1534 (2011), 1010.5827

[10] M. Cacciari, M. Greco, P. Nason, JHEP 9805, 007 (1998), hep-ph/9803400

[11] M. Cacciari, S. Frixione, N. Houdeau, M.L. Mangano, P. Nason et al., JHEP 1210, 137 (2012), 1205.6344

[12] R. Aaij et al. (LHCb collaboration), JHEP 1306, 064 (2013), 1304.6977

[13] J. Alves, A. Augusto et al. (LHCb collaboration), JINST 3, S08005 (2008)

[14] R. Aaij et al. (LHCb collaboration), Eur. Phys. J. C 71, 1645 (2011), 1103.0423

[15] R. Aaij et al. (LHCb collaboration), Eur. Phys. J. C 72, 2025 (2012), 1202. 6579

[16] R. Aaij et al. (LHCb collaboration), Eur. Phys. J. C 73, 2631. 27 p (2013)

[17] B. Abelev et al. (ALICE collaboration), Phys. Rev. Lett. 108, 082001 (2012), 1111.1630

[18] S. Chatrchyan et al. (CMS collaboration), JHEP 1202, 011 (2012), 1111.1557

[19] M. Butenschoen, B.A. Kniehl, Phys. Rev. D 84, 051501 (2011), 1105.0820

[20] M. Butenschoen, B.A. Kniehl, Phys. Rev. Lett. 106, 022003 (2011), 1009.5662

[21] J.M. Campbell, F. Maltoni, F. Tramontano, Phys. Rev. Lett. 98, 252002 (2007), hep-ph/0703113

[22] R. Aaij et al. (LHCb collaboration), Phys. Lett. B 718, 431 (2012), 1204.1462

[23] T. Sjöstrand, S. Mrenna, P.Z. Skands, JHEP 0605, 026 (2006), hep-ph/0603175

[24] R. Aaij et al. (LHCb collaboration) (2013), 1308.6729

[25] E. Ferreiro, F. Fleuret, J. Lansberg, A. Rakotozafindrabe, Phys. Rev. C 88, 047901 (2013), 1305.4569

[26] J. Albacete, N. Armesto, R. Baier, G. Barnafoldi, J. Barrette et al., Int. J. Mod. Phys. E 22, 1330007 (2013), 1301.3395

[27] F. Arleo, S. Peigne, JHEP 1303, 122 (2013), 1212.0434

[28] Y.Q. Ma, K. Wang, K.T. Chao, Phys. Rev. D 83, 111503 (2011), 1002.3987

[29] A. Likhoded, A. Luchinsky, S. Poslavsky (2013), 1305. 2389

[30] R. Aaij et al. (LHCb collaboration), JHEP 1310, 115 (2013), 1307.4285

[31] R. Aaij et al. (LHCb collaboration), Eur. Phys. J. C 73, 2631 (2013), 1307.6379

[32] M. Butenschoen, B.A. Kniehl, Nuclear Physics B - Proceedings Supplements 222-224, 151 (2012)

[33] B. Gong, L.P. Wan, J.X. Wang, H.F. Zhang, Phys. Rev. Lett. 110, 042002 (2013), 1205.6682

[34] K.T. Chao, Y.Q. Ma, H.S. Shao, K. Wang, Y.J. Zhang, Phys. Rev. Lett. 108, 242004 (2012), 1201.2675 